\title{
Extracting Foreground Masks towards Object Recognition
}

\author{
Amir Rosenfeld and Daphna Weinshall \\ School of Computer Science and Engineering \\ Hebrew university of Jerusalem, Israel 91904 \\ \{rosenfld, daphna\}ecs.huji.ac.il
}

\begin{abstract}
Effective segmentation prior to recognition has been shown to improve recognition performance. However, most segmentation algorithms adopt methods which are not explicitly linked to the goal of object recognition. Here we solve a related but slightly different problem in order to assist object recognition more directly - the extraction of a foreground mask, which identifies the locations of objects in the image. We propose a novel foreground/background segmentation algorithm that attempts to segment the interesting objects from the rest of the image, while maximizing an objective function which is tightly related to object recognition. We do this in a manner which requires no classspecific knowledge of object categories, using a probabilistic formulation which is derived from manually segmented images. The model includes a geometric prior and an appearance prior, whose parameters are learnt on the fly from images that are similar to the query image. We use graphcut based energy minimization to enforce spatial coherence on the model's output. The method is tested on the challenging VOCO9 and VOC10 segmentation datasets, achieving excellent results in providing a foreground mask. We also provide comparisons to the recent segmentation method of [7].
\end{abstract}

\section{Introduction}

Object recognition is one of the holy grails of computer vision. While many current object recognition methods do not rely on segmentation, a natural and common assumption is that good segmentation prior to recognition can improve the recognition results. This is because good segmentation is expected to narrow down the number of options to search among, allowing less room for false alarms and improving the run-time. In addition, a well segmented object will hopefully contain the relevant image features needed for recognition, thus reducing the signal to noise ratio [18].

Following this assumption, a segmentation algorithm is applied to the image, and the different segments are classified using a trained object classifier. The results may vary according to how accurate the segmentation is, and of course the quality of the classifier. However, the problem is that many popular segmentation algorithms [19, 10, 17], while having some desirable mathematical properties, have little to do with the end goal, which is recognizing objects. For instance, consider an image of a person's face. For the human observer, the person's hairline is not perceived as the boundary between the face and a different object. However, for an algorithm such as e.g. [10], the inter-segment distance is large. In order for the segmentation algorithm to keep the face and the hair in the same segment, the measurement of the distance between them has to be small enough. Thus, if segmentation was to achieve true object boundaries, it would have to ignore these differences somehow.

Why indeed should one expect a segmentation algorithm to identify true object boundaries? Most segmentation algorithms (e.g., [3, 10, 17]) are designed to segment the entire image with no regard to the notion of foreground or background. While this may create a good delineation of the boundaries of objects in the scene, the background becomes segmented as well. Thus we define a different goal - to directly extract a foreground mask from the image that indicates as accurately as possible where the objects lie within the scene. This can serve as a first step for an object recognition pipeline, without the additional noise of an over-segmented background and with the advantage of feeding the recognizer with relevant image regions.

In Section 3 we present a segregation algorithm that achieves figure-ground segmentation, with the intent of leaving the objects of interest whole and untouched. It appears that in order to distinguish whole objects from the background, one should have access to some implicit knowledge about the objects of interest. Our approach attempts to approximate this ideal situation, by training the 
foreground/background segmentation algorithm using other images similar to the query image, without using the classspecific knowledge.

We present a probabilistic formulation comprised of two components, corresponding to information about geometry (shape \& location) and appearance. The parameters of both priors are estimated separately for each query image, using images from a large pre-segmented training set. The geometric prior is estimated using the most similar images according to the GIST representation [16], while the appearance prior is estimated using the most similar images according to a Bag Of Words representation. In this way we avoid the daunting task of learning the entire probabilistic distribution of general foreground and background segments in images, relying instead on local approximations. Segmentation is achieved by solving an energy minimization problem over a superpixel graph attained using [1]. In Section 4 we describe experiments on images from the PASCAL 2009 and 2010 segmentation benchmarks, and offer a way to compare our results to that of [7].

\section{Related Work}

As the complexity of images in which we attempt to recognize objects keeps increasing, simple methods such as those based on Bag-of-Word features, which make a decision at an image level rather than object level, become less effective. Thus, much recent work on object recognition and multi-class labeling has focused on pre-segmenting the image before applying the recognition algorithms.

It has been shown in [14] that good spatial support is important for object categorization. They compared the performance of a classifier when presented with the visual features of the bounding box surrounding the object vs. the exact segmentation. For almost all object classes, exact segmentation improves the categorization accuracy. They used combinations of segments from over-segmented images using several popular segmentation methods, in order to find an optimal cover for the objects in question. Given the ground truth object boundary, the right combination of segments which cover it is found quite easily, but of course this information is not available for unseen images.

The work of [18] also shows how segmentation improves the performance of standard recognition algorithms. They first create a collection of segments by sampling the set of stable segmentations [17] out of a much larger set, created by varying the segmentation's parameters and choosing from among the results. The result is a "soup" of segments - many possibly overlapping segments covering the image. A baseline algorithm is applied to each of the resulting segments, thus ranking the segments according to classification confidence. Largely overlapping segments are removed and the top ranking ones are retained. While this segmentation algorithm shows some impressive results, it has a very long run time - many hours per image on a single computer, as reported by the authors. In addition the cost function optimized by the segmentation itself (i.e., stability of the segmentation) is not related to object recognition.

In [3], hierarchical segmentation is created using the results of a generic contour detector as input, achieving very good results. A different approach to ranking segments is described in [7]. They create a very large (up to ten thousands) set of segments and rank them based on a model trained to predict their plausibility as object segments, given a set of 34 regions and Gestalt properties.

ClassCut [2] automatically segments class objects, alternating between segmenting object instances and learning a class model. Their approach requires that the input set of images contain only a single class of images. Avoiding the question of perfect segmentation, [11] breaks the image into super-pixels and trains a classifier to differentiate between object classes based on the BOW representation of local features extracted from each super-pixel and its neighborhood. A CRF is employed to enforce spatial consistency resulting in a multi-label segmentation.

Some papers use training images to gain knowledge about the appearance of background and foreground. An elegant approach is presented in [4], who employ both topdown information (similar image fragments found in training images) and bottom up (image based) criteria to achieve impressive results. However, their dataset includes rather homogeneous (horse-side) images, while still requiring a substantial amount of training examples. In [13] they use multiple local and global image features types in a learning framework, in order to detect salient object in images. They train a CRF using these features, employing thousands of training images.

In contrast to such methods, some of which require many images for training, our approach is rather simple and uses only a few (well selected) training examples for each test image. Training is performed on the fly, requiring only a few seconds to segment the image and provide the foreground mask.

\section{Approach}

Given a set of manually segmented images, we wish to learn a model which will allow us to segment a novel image into two groups - foreground and background. The training data consists of a set $I_{1}, I_{2}, \ldots, I_{n}$ of images. For each image $I$ we are given some ground-truth pixel-wise labeling $L_{I}(x, y)$ for the objects in the image and their classes. The original labels belong to a set of categories: $L_{I}(x, y) \in$ $C=\left\{c_{1} \cdots c_{m}\right\}$, including a background category, which is typically labeled implicitly (i.e., no label).

The labels provide much useful information for the task. First, they provide the visual characteristics of foreground objects. Second, we observe the scene containing the ob- 
ject, making it possible to learn different segmentation rules for different scenes. Lastly, we are given geometric cues including shape and image location of foreground objects. Next we show how all of those cues combine into a single framework. Importantly, we do not use the label identity, only the foreground-background distinction.

Similarly to [11] we formulate the problem as graphpartitioning. Let $G=(V, E)$ denote a graph whose nodes correspond to superpixels. Those are obtained from [1], allowing us to control both the approximate size of the graph and its spatial regularity while still preserving object edges. Neighboring superpixels define the graph's edges.

Each node $v \in V$ is first assigned a category label $L(v) \in C$. Since we want to segment the image to foreground and background segments as opposed to creating class-specific labeling, we simplify this notation by using an indicator function $F_{v}$, which takes the value of 1 iff $L(v)$ is one of the foreground classes and 0 otherwise. Like $L(v)$, $F_{v}$ is well defined only if the superpixel contains exactly one label. Using fine enough superpixels, this is almost always true. Note that under this definition, objects which are adjacent in the image end up as one connected component of the resulting foreground mask.

\subsection{Probabilistic Formulation}

Our model assigns to each node $v \in V$ some probability that it belongs to the foreground object given the underlying superpixel $S_{v}, P\left(F_{v}=1 \mid S_{v}\right)$. It assigns to each pair of neighboring nodes $(u, v) \in E$ the probability that they have the same label (i.e, both are from the foreground objects or are background): $P\left(F_{u}=F_{v} \mid S_{u}, S_{v}\right)$. For brevity, we write

$$
\begin{gathered}
P_{f}(v)=P\left(F_{v}=1 \mid S_{v}\right) \\
P_{s}(u, v)=P\left(F_{u}=F_{v} \mid S_{u}, S_{v}\right)
\end{gathered}
$$

By doing so, we consider only two types of visual categories - foreground and background, avoiding the additional complexity of multi-class classification.

The two terms are combined to form the following energy function

$$
-\log P(F \mid G ; \alpha)=\sum_{v \in V} \eta_{f}(v)+\alpha \sum_{(u, v) \in E} \triangle_{s}(u, v)
$$

where $\alpha$ is a regularization parameter, controlling the tradeoff between the fidelity and smoothing terms. We show how to compute $\eta_{f}(v)=-\log P_{f}(v)$ in Section 3.2.2. $\triangle_{s}(u, v)$ is computed exactly as in [11], i.e.,

$$
\triangle_{s}(u, v)=-\log P_{s}(u, v)=\left(\frac{L\left(S_{u}, S_{v}\right)}{1+\left\|S_{u}-S_{v}\right\|}\right)\left[F_{i} \neq F_{j}\right]
$$

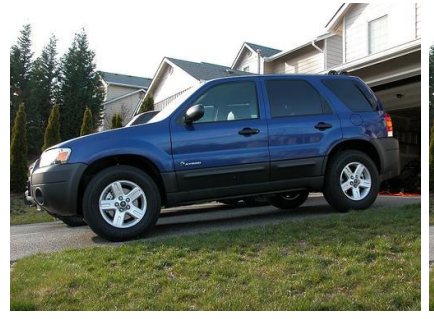

(a)

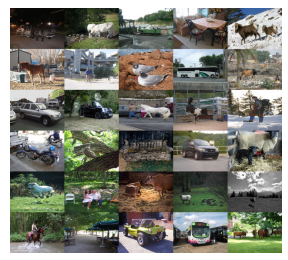

(c)

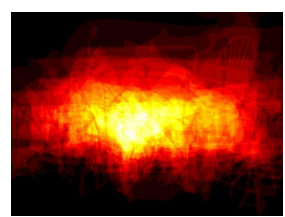

(f)

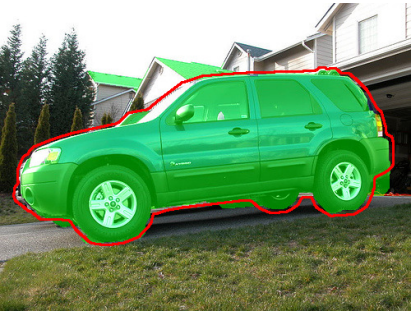

(b)

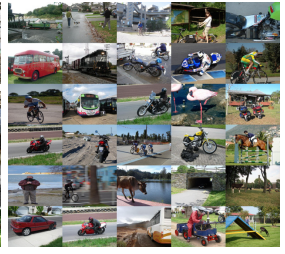

(d)

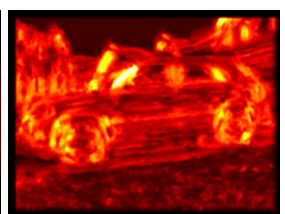

(g)

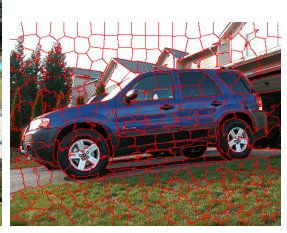

(e)

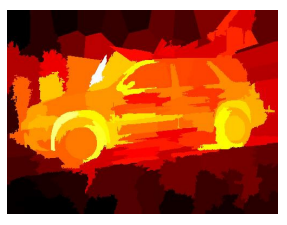

(h)
Figure 1. Automatic foreground extraction process: (a) Input image. (c) Geometrically similar images, used to train the geometric prior (f). (d) Visually similar images, used to train the appearance prior (g). (e) SLIC [1] superpixels, used to aggregate the priors, producing probability map (h). (b) Final foreground mask (green tint) with ground-truth overlaid (red borders).

where $L\left(S_{u}, S_{v}\right)$ denotes the length of the boundary between $S_{u}$ and $S_{v},\left\|S_{u}-S_{v}\right\|$ the norm of the color difference between superpixels in the LUV colorspace, and [.] the indicator function.

\subsection{Context on the fly}

The probability distribution in (1) may in reality be quite complex. In addition it is scene dependent, as a foreground object in one scene may very well have the local appearance properties of the background in another. We avoid the daunting task of learning a model to represent the entire distribution by simplifying the problem. Thus, for each test image we consider only similar images observed in the training set and use them to model the distribution. Our probability distribution is composed of two semi independent components: shape $\backslash$ location and local appearance. We use different training sets to learn each component independently. 


\subsubsection{Geometric Prior}

In order to obtain a suitable training set to learn the geometric prior, we use the GIST descriptor introduced in [16]. The descriptor gives low-dimensional representation of an image used to find similar images in very large datasets with good precision [8]. It sums up the response to different oriented Gabor filters over a grid of rectangular areas over the image, at multiple scales. The number of orientations may differ per scale. The responses are concatenated into a single feature vector. The standard implementation, used here, produces a feature vector of length 960 for a color image.

We compute the descriptor for all images in the training set. Given a test image $I$, we compute its GIST descriptor as well, denoted $\mathcal{G}(I) \in R^{G}$. We find the $K_{\mathcal{G}}$-nearest neighbors of this image from the set of training images, where distances are measured using the GIST representation of the images. Thus we retrieve a set of images whose general layout is similar to that of the query image. Denote those images by $n e i_{\mathcal{G}}(I)=\left\{I_{1}^{N}, \ldots, I_{K_{\mathcal{G}}}^{N}\right\}$ (see example in Fig. 1c).

The selected images are used to estimate a prior on the distribution of locations of foreground objects by summing over the foreground mask in each pixel $(\hat{x}, \hat{y})$ :

$$
P_{f}^{\mathcal{G}}(\hat{x}, \hat{y})=\frac{1}{K_{\mathcal{G}}} \sum_{I_{j}^{N} \in n e i_{\mathcal{G}}(I)}\left[L_{I_{j}^{N}}(\hat{x}, \hat{y}) \in \text { foreground }\right]
$$

where $L_{I}(\hat{x}, \hat{y})$ denotes the label of the image $I$ at the coordinates $(x, y)$ after being normalized to the size of the test image, and [.] denotes the indicator function. $P_{f}^{\mathcal{G}}(\hat{x}, \hat{y})$ is smoothed using a Gaussian kernel with $\sigma=7$, to produce a more continuous result. Thus we obtain a pixel-wise probability for the presence of a foreground object, see Fig. 1f.

Note that this prior is quite informative, since we sum over the objects' masks and not only their centers, thus gaining information about the outline of the objects of interest. We call this the geometric prior; examples are shown in Fig. 2. The figure also shows the advantage of computing this prior from Gist-based nearest neighbors, rather than using a generic mask computed from all the images in the database (or a random sample of the images).

\subsubsection{Appearance Prior}

The GIST descriptor, while enabling the retrieval of scenes with similar layout, typically retrieves images with different scene content than the query image (see Fig. 2). Thus if we wish to discriminate between foreground and background on the basis of visual features, we need to choose a different learning set. In order to find scenes with similar content, we use a standard BOW method: a $k$-means dictionary is computed for a large set of local descriptors from the set of training images.

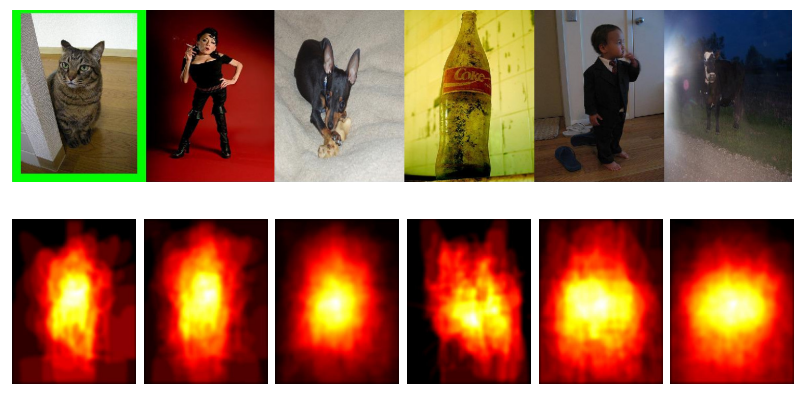

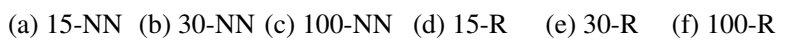
Figure 2. Geometric Prior: Using scenes of similar layout (not necessarily similar objects), object location and shape are estimated. Top: green outline marks the query image, followed by its 5 nearest GIST neighbors. Bottom: Location priors (normalized) are calculated by summing up different object masks from a set of retrieved images. NN marks the masks computed from the 15, 30 and 100 Nearest Neighbor images, and $\mathbf{R}$ those masks computed from Random images. Clearly similar images consistently generate better geometric priors.

Thus, for each training image $I$ we store the $L_{1}$-normalized and tf-idf weighted histogram of visual word appearances. Denote this appearance descriptor by $\mathcal{A}(I)$. Given a test image, we find its $K_{\mathcal{A}}$ nearest neighbors from the set of training images where distances are computed using the BOW representation of the images. Denote those images by $n e i_{\mathcal{A}}(I)=\left\{I_{1}^{N}, \ldots, I_{K_{\mathcal{A}}}^{N}\right\}$. They are used to estimate the prior on the visual properties of foreground and background objects, as described in the next section.

Our goal is to assign a foreground probability to each pixel. Let $\widetilde{P}$ denote the set of pixels belonging to the images in the set $n e i_{\mathcal{A}}(I)$. For pixel $p$, let $F_{p}$ denote the indicator function defined over pixels: $F_{p}=\left[L_{I}(x, y) \in\right.$ foreground]. The positive and negative examples for foreground pixels are

$$
\begin{aligned}
& \widetilde{P}_{F}=\left\{p \in \widetilde{P}: F_{p}=1\right\} \\
& \widetilde{P}_{B}=\left\{p \in \widetilde{P}: F_{p_{i}}=0\right\}
\end{aligned}
$$

In order to make fast segmentation possible despite the fact that $\widetilde{P}$ is different for each image, we use an approximation of the Parzen-window estimator. Let $I_{t}$ be a test image. We define a dense regular grid (every 2 pixels) on $I_{t}$. Let $p \in P_{I_{t}}$ be a pixel in this grid denoted $P_{I_{t}}$. Define $P_{f}^{+}(p)$ to be the estimator of the probability that $p$ is drawn from the positive sample distribution

$$
P_{f}^{+}(p)=\sum_{q \in \widetilde{P}_{F}} \phi(p, q)
$$

with kernel function $\phi(p, q) \propto e^{-(d(p)-d(q))^{2} / 2 \sigma^{2}}$, and $d(p) \in \mathcal{R}^{D}$ denotes the feature vector for the local descrip- 
tor at pixel $p$. Similarly

$$
P_{f}^{-}(p)=\sum_{q \in \widetilde{P}_{B}} \phi(p, q)
$$

The probability $P_{f}(p)$ is thus calculated by the normalized ratio:

$$
P_{f}(p)=\frac{P_{f}^{+}(p)}{P_{f}^{+}(p)+P_{f}^{-}(p)}
$$

This is done for each $p \in P_{I_{t}}$.

Since we use an exponential kernel function, the density can be well approximated by considering only the k-nearest neighbors of $d(p)$ in the set of training examples. Although nearest-neighbor algorithms are constantly improving [15], it is still too time consuming if we want reasonable performance: we need to perform $\left|P_{I_{t}}\right|$ searches in the sample set of size $|\widetilde{P}|$, which can be quite large (millions).

Instead, we create a quantized code-book (as in the bagof-features) model. Adapting the framework described in [21], PHOW Descriptors are sampled at multiple scales and locations from the training images. The descriptors are quantized via $K$-means clustering. Each local descriptor in both the test and training set is assigned its nearest cluster in the $K$-means dictionary. Let $w_{v} \in \mathbf{W}=\left\{w_{1}, \ldots, w_{k}\right\}$ denote the visual word assigned to the descriptor of pixel $p$. The probability of foreground for the pixel $p$ (Eq. (6)) is approximated by the probability for the corresponding visual word $w_{v}$ :

$$
\tilde{P}_{f}\left(w_{p}\right)=\frac{\sum_{q \in \widetilde{P}_{F}}\left[w_{q}=w_{p}\right]}{\sum_{q \in \widetilde{P}_{F}}\left[w_{q}=w_{p}\right]+\sum_{q \in \widetilde{P}_{B}}\left[w_{q}=w_{p}\right]}
$$

In other words, we count how many times each visual word is assigned the foreground or background labels.

This approximation allows us to quantize once the local descriptors of each of the training and test images. During run-time, the density function (7) is estimated by counting features from the corresponding images, which is very quick and requires little memory. In the end, each pixel in the grid $p \in P_{I_{t}}$ is assigned a visual word $w_{p}$ and a probability $\tilde{P}_{f}\left(w_{p}\right)$.

\subsection{Object-Edge Preserving Smoothing}

In order to obtain the probabilities $P_{f}(v)$, we aggregate the local probabilities from both geometric prior (5) and appearance prior (7) using the superpixels as local decision boundaries. Let $S_{v}$ denote the set of pixels in the area of the superpixel $v$, then

$$
P_{f}(v)=\frac{1}{\left|S_{v}\right|} \sum_{p \in S_{v}} \tilde{P}_{f}\left(w_{p}\right)\left(P_{f}^{\mathcal{G}}(\hat{x}, \hat{y})\right)^{\gamma}
$$

for $w_{p}$ the visual word assigned to the descriptor of pixel $p$, and $p=(\hat{x}, \hat{y})$.

The non-uniform smoothing resulting from the use of super-pixels has the advantage of enhancing the effect of $\tilde{P}_{f}\left(w_{p}\right)$ and $P_{f}^{\mathcal{G}}(\hat{x}, \hat{y})$ inside objects and arbitrarily dispersing the energy of those in more uniform image areas. This is repeated for two levels of superpixel granularity - coarse and fine. Coarse superpixels are used to estimate $P_{f}(v)$ as in Eq. (8). Then each image pixel in $S_{v}$ is assigned the probability $P_{f}(v)$ and the process is repeated, only this time computing $P_{f}(v)$ over a more finely segmented image.

The coarse stage aggregates probabilities from relatively large areas, thus potentially capturing more informative local features at the cost of reduced accuracy. The fine superpixels allow for larger flexibility in the final segmentation stage, since they are used as the graph nodes in the optimization process, after Eqs. (7) and (4) are plugged into Eq. (3). The energy is minimized using the graph-cut optimization of $[12,11,6,5]$. The parameter $\alpha$ is determined by optimizing over a small portion $(10 \%)$ of the dataset, and is kept constant at 16 throughout the experiments. It can also be chosen by cross-validation of the training data. The algorithm is summarized in the box below.

\section{Experiments}

The algorithm was tested extensively on the Pascal VOC09 and VOC10 datasets [9], which have many training images annotated with manual segmentation. We computed local image descriptors on a dense regular grid (with 2-pixel spacing) using the color-PHOW implementation of [21], which is computed at multiple scales. We summed up the foreground prior estimated for each scale independently with uniform weighting. The choice of descriptor was motivated by the survey of [20]. The size of the visual dictionary was set to $K=1000$. We computed the GIST descriptors on a $4 \times 4$ grid for all training images using a slightly modified version of [16], allowing us to deal with rectangular (rather than square) images. After the preprocessing stage of extracting and quantizing dense local descriptors, the runtime of the algorithm is 1-3 seconds on a PC with $8 \mathrm{~Gb}$ of RAM and Intel core i5 CPU.

Examples can be seen in Fig. 3 and the suppl. material. Clearly, the algorithm deals well with background clutter and multiple connected components. The code will be made available on the web.

\subsection{Appearance vs. Geometry}

To evaluate the contribution of the appearance and geometric priors separately, we treat the problem as that of classification. Varying the threshold on the probability maps obtained by using either the appearance prior or the geometric prior alone (or their combination), we obtain a precisionrecall curve on the test dataset - see Fig. 4. Perhaps sur- 


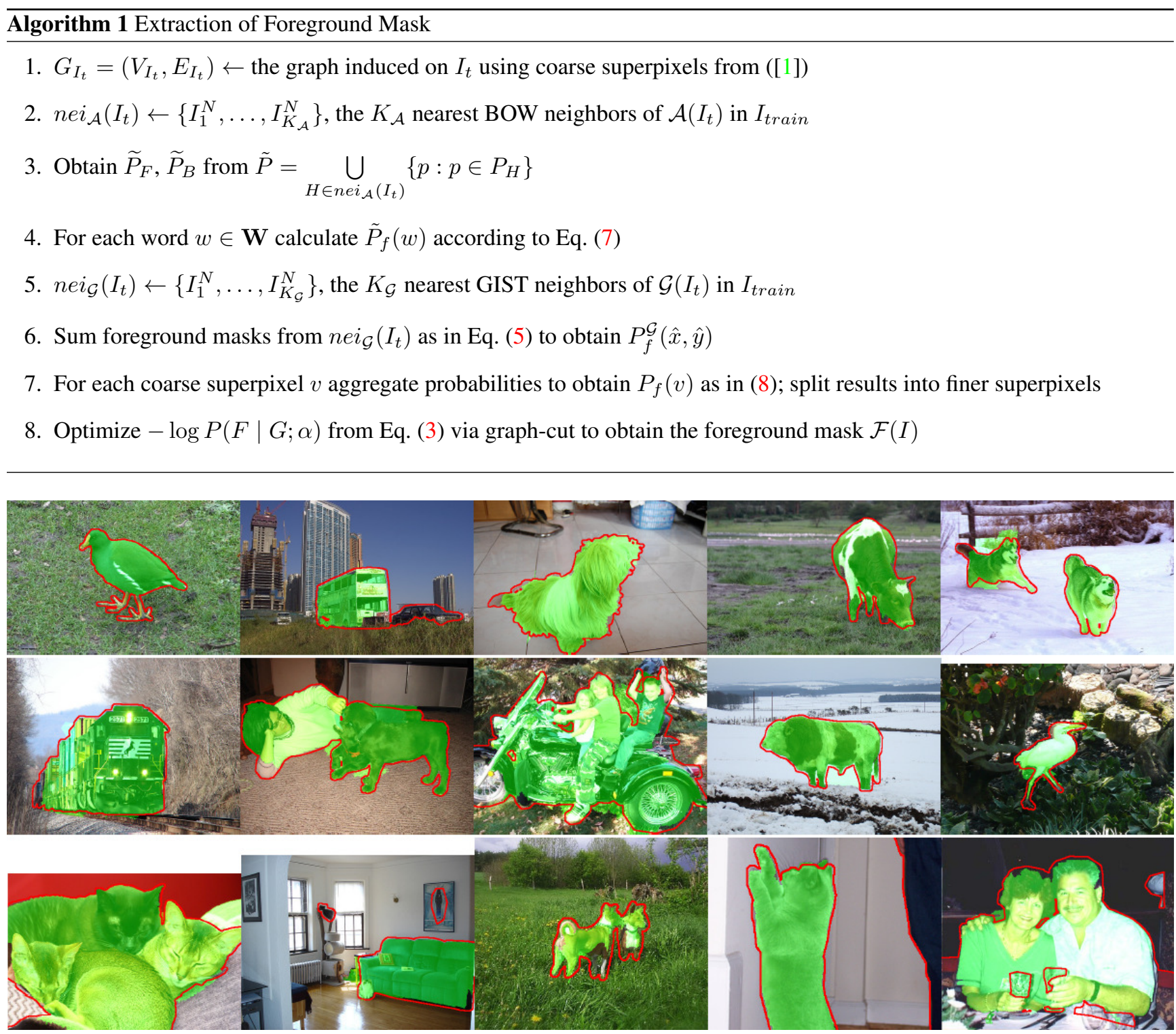

Figure 3. Some of our segmentation results on VOC09 and VOC10. The algorithm provides a foreground mask (green tint) with the goal to extract all foreground objects (red outline), without oversegmenting the background. All results were obtained using the same parameters of $K_{\mathcal{A}}=K_{\mathcal{G}}=30$ along with the geometric prior. The foreground need not be a single connected component (1st row, right). The algorithm succeeds in highly cluttered backgrounds ( 2 nd row, right $\&$ center). See supp. material for many more examples.

prisingly geometry alone is a stronger cue than local appearance. Arguably, this happens because most objects are approximately centered in the PASCAL benchmarks. However, the learning process also contributes to this success; as can be seen in Fig. 2, both location and shape are captured more concisely when using good training examples.

\subsection{Quality of Segmentation}

\subsubsection{Overlap score}

Since our algorithm provides a foreground mask (sometime with several connected components), we score each result produced by our algorithm according to its overlap score:

$$
O=\frac{\left|S \cap S^{\prime}\right|}{\left|S \cup S^{\prime}\right|}
$$

where $S$ is the ground-truth foreground mask, i.e., the union of all object segments, and $S^{\prime}$ is the result of our algorithm. This scoring scheme reflects the fact that our algorithm makes no effort to split adjacent foreground objects. Instead, it aims to produce foreground masks that include all objects in the image. The average of this score over the test set of the VOC09 benchmark is given in Table 1. 


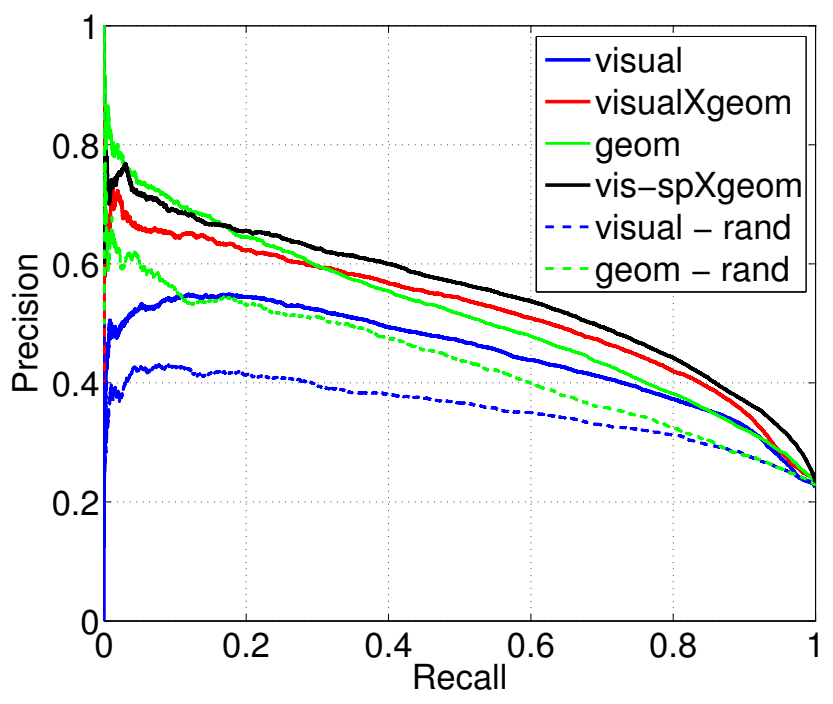

Figure 4. Performance comparison of appearance and geometrical priors. Geometry alone (solid green) is stronger than local appearance cues (solid blue). Superpixel aggregation (black) further enhances the result, for all but low-recall cases. Dashed green/blue lines show the performance drop when choosing random images for learning instead of those chosen by our method.

\begin{tabular}{|c|c|c|c|}
\hline Dataset & CPMC & Ours & Ours-Mask \\
\hline VOC09 & 0.4018 & 0.4263 & 0.4708 \\
\hline VOC10 & - & 0.4107 & 0.4570 \\
\hline
\end{tabular}

Table 1. Comparison of our method's overlap score to CPMC [7], using their first ranked segmentation. In the column under "OursMask" we give the modified score, where image adjacent object segments in the ground-truth are merged into one connected component.

To study the effect of the algorithm's parameters $K_{\mathcal{G}}$ and $K_{\mathcal{A}}$, we varied them and calculated the overlap score over the test set. We tested how switching on and off the use of geometric prior changed the final results. Summary of this test can be seen in Fig. 5. Clearly the use of the geometric prior isn't always the right choice, as can be seen by the mean grade attained by choosing for each image its best scoring method. To illustrate, Fig. 6 shows a case where using the geometric prior hinders the result.

\subsubsection{Comparison to Segmentation}

The following score, used in [7], measures the covering of the ground-truth segmentation by a machine segmentation:

$$
C\left(S, S^{\prime}\right)=\frac{1}{N} \sum_{R \in S}|R| * \max _{R^{\prime}} O\left(R, R^{\prime}\right)
$$

where $N$ denotes the number of pixels in the image, $|R|$ the number of pixels in the ground truth segment, and $O$ the overlap.

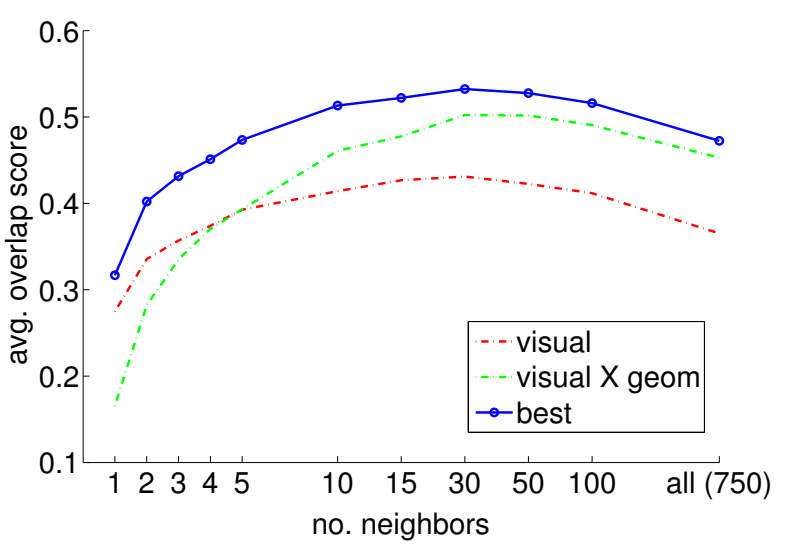

Figure 5. Mean segment overlap score on VOC09 for varying numbers of nearest neighbors chosen for learning geometric \& appearance priors $\left(K_{\mathcal{A}}=K_{\mathcal{G}}\right)$. Dashed red lines ("visual") - performance using the appearance prior only. Green ("visual x geom") contribution of the geometric prior. The solid blue curve ("best") shows performance when choosing for each image its best scoring method.

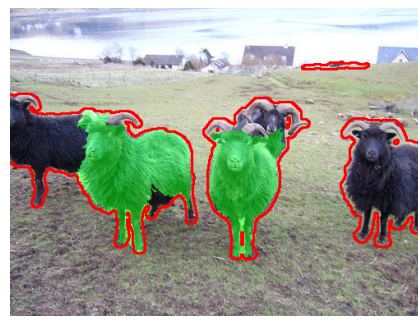

(a)

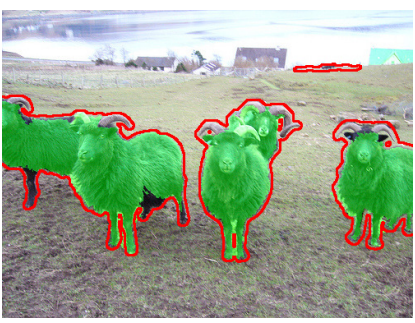

(b)
Figure 6. Geometric prior doesn't always help. (a) Segmentation using appearance \& geometric prior. (b) Segmentation using appearance only.

We compare our results to those of [7], which achieves excellent segmentation results by creating diverse segmentations and ranking them automatically using a learnt model. Having produced multiple segmentations, they compute the average of the best covering score for varying number of segments, chosen according to their ranking.

The mean covering score we obtain for a single segmentation using $K_{\mathcal{A}}=K_{\mathcal{G}}=30$ is 0.4263 . On average this is slightly better when compared to the score obtained by the first ranking segment of [7], which is 0.4018. With additional segments (which our method does not produce), their method achieves higher accuracies than ours. We note that the runtime of [7] is approx. 3 minutes, as compared to 1-3 seconds of our own method; this is because their algorithm solves the more complicated problem of full segmentation, and does so many times.

Since our algorithm aims at creating whole foreground masks, it is at a disadvantage when the scoring method ex- 
pects it to split connected foreground blobs into different segments, as was done in the comparison above. A more suitable score should regard connected foreground objects as the same ground-truth segment. Under this relaxation, we achieve a much higher result of 0.4708 . The results are summarized in Table 1, where results on VOC10 are reported as well.

\section{Discussion \& Conclusions}

We have presented an efficient and effective algorithm for foreground/background segmentation, motivated by object recognition perspective. The algorithm learns both geometric and appearance priors for the task. For each prior, a different set of training images is chosen independently, in order to maximize the relevant data in the training set. This choice allows for learning from limited datasets, as images whose content and layout are both similar to the query image may be rare. It leads to a powerful representation that seems to discriminate foreground from background quite well. We note that although the ground-truth annotations of the dataset contain rich class-specific information on multiple classes, none of this information is used by our algorithm, none but the distinction between background and foreground. The algorithm was tested on two challenging datasets, Pascal VOC09 and VOC10.

To assist object recognition, the foreground mask computed by our algorithm can be fed into any choice of recognition algorithm. New features may be subsequently computed more robustly from the foreground area only, before attempting final recognition.

\section{References}

[1] R. Achanta, A. Shaji, K. Smith, A. Lucchi, P. Fua, and S. Susstrunk. SLIC Superpixels. Technical report, EPFL, 2010. 2, 3, 6

[2] B. Alexe, T. Deselaers, and V. Ferrari. Classcut for unsupervised class segmentation. Computer Vision-ECCV 2010, pages 380-393, 2010. 2

[3] P. Arbeláez, M. Maire, C. Fowlkes, and J. Malik. From contours to regions: An empirical evaluation. 2009. 1, 2

[4] E. Borenstein. Combining top-down and bottom-up segmentation. In Proceedings IEEE workshop on Perceptual Organization in Computer Vision, CVPR, 2004. 2

[5] Y. Boykov and V. Kolmogorov. An experimental comparison of min-cut/max-flow algorithms for energy minimization in vision. IEEE transactions on Pattern Analysis and Machine Intelligence, 26(9):1124-1137, September 2004. 5

[6] Y. Boykov, O. Veksler, and R. Zabih. Efficient approximate energy minimization via graph cuts. IEEE transactions on Pattern Analysis and Machine Intelligence, 20(12):12221239, November 2001. 5

[7] J. Carreira and C. Sminchisescu. Constrained parametric min-cuts for automatic object segmentation. In Computer
Vision and Pattern Recognition (CVPR), 2010 IEEE Conference on, pages 3241-3248. IEEE, 2010. 1, 2, 7

[8] M. Douze, H. Jégou, H. Sandhawalia, L. Amsaleg, and C. Schmid. Evaluation of gist descriptors for web-scale image search. In Proceeding of the ACM International Conference on Image and Video Retrieval, pages 1-8. ACM, 2009. 4

[9] M. Everingham, L. Van Gool, C. Williams, J. Winn, and A. Zisserman. The PASCAL visual object classes (VOC) challenge. International journal of computer vision, 88(2):303-338, 2010. 5

[10] P. F. Felzenszwalb and D. P. Huttenlocher. Efficient GraphBased Image Segmentation. International Journal of Computer Vision, 59(2):167-181, Sept. 2004. 1

[11] B. Fulkerson, A. Vedaldi, and S. Soatto. Class segmentation and object localization with superpixel neighborhoods. In Proceedings of the International Conference on Computer Vision, October 2009. 2, 3, 5

[12] V. Kolmogorov and R. Zabih. What energy functions can be minimized via graph cuts? IEEE transactions on Pattern Analysis and Machine Intelligence, 26(2):147-159, February 2004. 5

[13] T. Liu, Z. Yuan, J. Sun, J. Wang, N. Zheng, X. Tang, and H. Shum. Learning to detect a salient object. Published by the IEEE Computer Society, 2010. 2

[14] T. Malisiewicz and A. Efros. Improving spatial support for objects via multiple segmentations. 2, 2007. 2

[15] M. Muja and D. G. Lowe. Fast approximate nearest neighbors with automatic algorithm configuration. In International Conference on Computer Vision Theory and Application VISSAPP'09), pages 331-340. INSTICC Press, 2009. 5

[16] A. Oliva and A. Torralba. Modeling the shape of the scene: A holistic representation of the spatial envelope. International Journal of Computer Vision, 42(3):145-175, 2001. 2, 4, 5

[17] A. Rabinovich, T. Lange, J. Buhmann, S. Belongie, and E. Zurich. Model order selection and cue combination for image segmentation. CVPR (1), pages 1130-1137, 2006. 1, 2

[18] A. Rabinovich, A. Vedaldi, and S. Belongie. Does image segmentation improve object categorization. 1, 2

[19] J. Shi and J. Malik. Normalized Cuts and Image Segmentation. Analysis, 22(8):888-905, 2000. 1

[20] K. E. A. van de Sande, T. Gevers, and C. G. M. Snoek. Evaluating color descriptors for object and scene recognition. IEEE Transactions on Pattern Analysis and Machine Intelligence, 32(9):1582-1596, 2010. 5

[21] A. Vedaldi and B. Fulkerson. VLFeat: An open and portable library of computer vision algorithms. http://www . vlfeat.org/, 2008. 5 